UT-967

September 2001

\title{
Lattice Chiral Symmetry and the Wess-Zumino Model
}

\author{
Kazuo Fujikawa and Masato Ishibashi \\ Department of Physics, University of Tokyo \\ Bunkyo-ku, Tokyo 113, Japan
}

\begin{abstract}
A lattice regularization of the supersymmetric Wess-Zumino model is studied by using Ginsparg-Wilson operators. We recognize a certain conflict between the lattice chiral symmetry and the Majorana condition for Yukawa couplings, or in Weyl representation a conflict between the lattice chiral symmetry and Yukawa couplings. This conflict is also related, though not directly, to the fact that the kinetic (Kähler) term and the superpotential term are clearly distinguished in the continuum Wess-Zumino model, whereas these two terms are mixed in the GinspargWilson operators. We illustrate a case where lattice chiral symmetry together with naive Bose-Fermi symmetry is imposed by preserving a SUSY-like symmetry in the free part of the Lagrangian; one-loop level non-renormalization of the superpotential is then maintained for finite lattice spacing, though the finite parts of wave function renormalization deviate from the supersymmetric value. All these properties hold for the general Ginsparg-Wilson algebra independently of the detailed construction of lattice Dirac operators.
\end{abstract}

\section{Introduction}

Supersymmetry is a result of the pursuit of maximal symmetry between bosons and fermions, and it exhibits remarkable dynamical properties [1]. A study of the simplest model due to Wess and Zumino[2] led to the so-called non-renormalization theorem both in the component approach [3] and in superfield formulation[四. As was emphasized in the first paper in [4], the potential part of the model is free of any (even finite) renormalization up to all orders in perturbation when renormalized at vanishing momenta. However, it may be fair to say that a satisfactory regularization of general supersymmetry, in particular in component formulation, is still missing.

On the other hand, we recently witnessed a remarkable progress in lattice gauge theory. A basic algebraic relation which lattice Dirac operators should satisfy was clearly stated by Ginsparg and Wilson[0], and an explicit solution to the algebra was given [6]. This solution exhibits quite interesting chiral properties [7] [8] [9] including locality properties [10] [11] [12]; the lattice Dirac operator is no more ultra-local but exponentially local. See, for example, Ref. [13] for reviews of this development. 
It is thus interesting to see what we learn about the regularization of supersymmetry if one uses the Ginsparg-Wilson algebra. In this paper, we study this problem by using the simplest Wess-Zumino model. There already exist several papers on this subject 14 15], but our approach differs from those studies in several essential aspects. Our interest is in the implications of lattice chiral symmetry satisfied by the Ginsparg-Wilson operators, and we study the divergence cancellation by perturbative calculations, setting aside the basic issue of supersymmetry algebra 14] 15. The perturbative lattice analysis of Yukawa couplings is expected to be important when one applies a non-perturbative analysis to the gauge theoretical sector of a supersymmetric model of elementary particles, just to preserve the consistency of various sectors of a single model.

We show that there exists a conflict between the lattice chiral symmetry and the Majorana condition for Yukawa couplings, or in Weyl representation a conflict between the lattice chiral symmetry and Yukawa couplings. This conflict is also related, though not directly, to the fact that the kinetic (Kähler) term and the superpotential term are clearly distinguished in the continuum Wess-Zumino model (in a symbolic superfield notation 四)

$$
S=\int \phi^{\dagger} \phi+\int\left(m \phi^{2}+g \phi^{3}\right)+h . c .,
$$

whereas these two terms are mixed in the Ginsparg-Wilson operators, as is explained later. We then illustrate in some detail a case where lattice chiral symmetry together with naive Bose-Fermi symmetry is imposed by sacrificing the precise Majorana condition but by preserving a SUSY-like symmetry in the free part of the Lagrangian. One-loop level nonrenormalization of the superpotential is shown to be maintained for finite lattice spacing, though the finite parts of wave function renormalization deviate from the supersymmetric value.

A pioneering work of the lattice regularization of supersymmetry was initiated by Dondi and Nicolai [16]. Among the past works, our analysis is closely related to the work

of Bartels and Kramer 17]. See also the early works on the Wess-Zumino model 18 and supersymmetric gauge theory [19]. As for the recent analyses of lattice regularization of supersymmetric gauge theory on the basis of the Ginsparg-Wilson operator (or the domain-wall fermion), see papers in 20.

\section{Brief Summary of the Ginsparg-Wilson Operators}

We are interested in the implications of the Ginsparg-Wilson algebra per se independently of the detailed properties of lattice Dirac operators. To be more precise, we analyze the implications of the general algebraic relation 21

$$
\gamma_{5}\left(\gamma_{5} D\right)+\left(\gamma_{5} D\right) \gamma_{5}=2 a^{2 k+1}\left(\gamma_{5} D\right)^{2 k+2}
$$

where $D$ is the lattice Dirac operator and the parameter $a$ is the lattice spacing; $k$ stands for non-negative integers, and $k=0$ corresponds to the conventional Ginsparg-Wilson relation. When one defines a hermitian operator $H$ by

$$
H=a \gamma_{5} D=H^{\dagger}=a D^{\dagger} \gamma_{5}
$$


the above algebraic relation is written as

$$
\gamma_{5} H+H \gamma_{5}=2 H^{2 k+2}
$$

We can also show

$$
\gamma_{5} H^{2}=\left(\gamma_{5} H+H \gamma_{5}\right) H-H\left(\gamma_{5} H+H \gamma_{5}\right)+H^{2} \gamma_{5}=H^{2} \gamma_{5}
$$

which implies

$$
H^{2}=a^{2} D^{\dagger} D=\gamma_{5} H^{2} \gamma_{5}=a^{2} D D^{\dagger}
$$

When we define

$$
\begin{aligned}
& \Gamma_{5} \equiv \gamma_{5}-H^{2 k+1}, \\
& \hat{\gamma}_{5} \equiv \gamma_{5}-2 H^{2 k+1},
\end{aligned}
$$

the defining algebra (1.2) is written as

$$
\Gamma_{5} H+H \Gamma_{5}=0
$$

and

$$
\left(\hat{\gamma}_{5}\right)^{2}=1
$$

We can also show the relation

$$
\gamma_{5} \Gamma_{5}+\Gamma_{5} \gamma_{5}=2 \Gamma_{5}^{2}=2\left(1-H^{4 k+2}\right)
$$

which implies $H^{2} \leq 1$. We next note 13

$$
D=P_{+} D \hat{P}_{-}+P_{-} D \hat{P}_{+}
$$

or

$$
H=P_{+} H \hat{P}_{-}+P_{-} H \hat{P}_{+}
$$

which implies

$$
\begin{aligned}
& P_{+} H=P_{+} H \hat{P}_{-}=H \hat{P}_{-}, \\
& P_{-} H=P_{-} H \hat{P}_{+}=H \hat{P}_{+} .
\end{aligned}
$$

Here we defined two projection operators

$$
\begin{aligned}
& P_{ \pm}=\frac{1}{2}\left(1 \pm \gamma_{5}\right) \\
& \hat{P}_{ \pm}=\frac{1}{2}\left(1 \pm \hat{\gamma}_{5}\right)
\end{aligned}
$$

which satisfy the relations

$$
\begin{aligned}
& P_{+} \hat{P}_{+}=P_{+} \Gamma_{5}, \\
& P_{-} \hat{P}_{-}=-P_{-} \Gamma_{5} .
\end{aligned}
$$


The following relations are often used in our calculation

$$
\begin{aligned}
& P_{ \pm} \Gamma_{5} H=-H \Gamma_{5} P_{\mp}=\Gamma_{5} H P_{\mp}, \\
& P_{ \pm} \Gamma_{5} H P_{ \pm}=0
\end{aligned}
$$

which follow from $P_{+} H-H P_{-}=H^{2 k+2}$ and $P_{+}+P_{-}=1$.

We then define the chiral components 13

$$
\bar{\psi}_{L, R}=\bar{\psi} P_{ \pm}, \quad \psi_{R, L}=\hat{P}_{ \pm} \psi
$$

and the scalar and pseudscalar densities by[22]

$$
\begin{aligned}
& S(x)=\bar{\psi}_{L} \psi_{R}+\bar{\psi}_{R} \psi_{L}=\bar{\psi} \gamma_{5} \Gamma_{5} \psi, \\
& P(x)=\bar{\psi}_{L} \psi_{R}-\bar{\psi}_{R} \psi_{L}=\bar{\psi} \Gamma_{5} \psi .
\end{aligned}
$$

An explicit form of $H$ in momentum representation, which is local and free of species doublers, is given by 23] 24]

$$
\begin{aligned}
H\left(a p_{\mu}\right) & =\gamma_{5}\left(\frac{1}{2}\right)^{\frac{k+1}{2 k+1}}\left(\frac{1}{\sqrt{H_{W}^{2}}}\right)^{\frac{k+1}{2 k+1}}\left\{\left(\sqrt{H_{W}^{2}}+M_{k}\right)^{\frac{k+1}{2 k+1}}-\left(\sqrt{H_{W}^{2}}-M_{k}\right)^{\frac{k}{2 k+1}} \frac{s^{\prime}}{a}\right\} \\
& =\gamma_{5}\left(\frac{1}{2}\right)^{\frac{k+1}{2 k+1}}\left(\frac{1}{\sqrt{F_{(k)}}}\right)^{\frac{k+1}{2 k+1}}\left\{\left(\sqrt{F_{(k)}}+\tilde{M}_{k}\right)^{\frac{k+1}{2 k+1}}-\left(\sqrt{F_{(k)}}-\tilde{M}_{k}\right)^{\frac{k}{2 k+1}} s\right\}
\end{aligned}
$$

where

$$
\begin{aligned}
F_{(k)} & =\left(s^{2}\right)^{2 k+1}+\tilde{M}_{k}^{2} \\
\tilde{M}_{k} & =\left[\sum_{\mu}\left(1-c_{\mu}\right)\right]^{2 k+1}-m_{0}^{2 k+1}
\end{aligned}
$$

and

$$
\begin{aligned}
& s_{\mu}=\sin a p_{\mu} \\
& c_{\mu}=\cos a p_{\mu} \\
& \mathcal{s}=\gamma^{\mu} \sin a p_{\mu} .
\end{aligned}
$$

For $k=0$, this operator is reduced to Neuberger's overlap operator [6]. Our Euclidean Dirac matrices are anti-hermitian, $\left(\gamma^{\mu}\right)^{\dagger}=-\gamma^{\mu}$, but the inner product is defined to be $s^{2} \geq 0$. Note that $H^{2}$ (and consequently $\Gamma_{5}^{2}$ ) is independent of Dirac matrices $\square$. The parameter $m_{0}$ is constrained by $0<m_{0}<2$, and $2 m_{0}^{2 k+1}=1$ gives a proper normalization of $H$, namely, for an infinitesimal $p_{\mu}$, i.e., for $\left|a p_{\mu}\right| \ll 1$,

$$
H \simeq-\gamma_{5} a \not p\left(1+O(a p)^{2}\right)+\gamma_{5}\left(\gamma_{5} a \not p\right)^{2 k+2}
$$

to be consistent with $H=\gamma_{5} a D$; the last term in the rigth-hand side is the leading term of chiral symmetry breaking terms.

Most of our calculations in the following are independent of the detailed construction of $H$.

\footnotetext{
${ }^{1}$ The basic algebra implies $\left[\gamma_{5}, H^{2}\right]=0$ and thus $H^{2}$ contains an even number of Dirac matrices. We thus expect that free $H^{2}$ is generally independent of Dirac matrices since it depends on a single momentum and parity conserving.
} 


\section{Lattice Chiral Symmetry and the Majorana Con- dition}

The continuum Wess-Zumino model is defined by[3]

$$
\begin{aligned}
\mathcal{L}= & \frac{1}{2} \chi^{T} C i \not \partial \chi+\frac{1}{2} m \chi^{T} C \chi+g \chi^{T} C\left(P_{+} \phi P_{+}+P_{-} \phi^{\dagger} P_{-}\right) \chi \\
& +\phi^{\dagger} \partial_{\mu} \partial^{\mu} \phi+F^{\dagger} F+m\left[F \phi+(F \phi)^{\dagger}\right]+g\left[F \phi^{2}+\left(F \phi^{2}\right)^{\dagger}\right]
\end{aligned}
$$

where $\chi$ is a Euclidean Majorana fermion and

$$
\phi=(A+i B) / \sqrt{2} .
$$

The variable $F$ stands for a complex auxiliary field. There exists no Euclidean Majorana fermion in a strict sense, and we follow the conventional treatment of a Euclidean Majorana fermion [25]. See Ref.[26] for a nice account of Euclidean Majorana fermions. It is easier to handle a lattice Dirac fermion than a lattice Majorana fermion. We thus start with a lattice Dirac fermion with Yukawa couplings, and later we discuss the reduction of the Dirac fermion to a Majorana fermion.

\subsection{Dirac fermions and Yukawa couplings}

We can think of 3 different Lagrangians for the Dirac fermions with Yukawa couplings. The first one is the most natural one consistent with lattice chiral symmetry, which is softly broken by the mass term,

$$
\begin{aligned}
\mathcal{L}_{(1)}= & \bar{\psi} D \psi+m \bar{\psi} \gamma_{5} \Gamma_{5} \psi+2 g \bar{\psi}\left(P_{+} \phi \hat{P}_{+}+P_{-} \phi^{\dagger} \hat{P}_{-}\right) \psi \\
= & \bar{\psi}_{R} D \psi_{R}+\bar{\psi}_{L} D \psi_{L}+m\left[\bar{\psi}_{R} \psi_{L}+\bar{\psi}_{L} \psi_{R}\right] \\
& +2 g\left[\bar{\psi}_{R} \phi \psi_{L}+\bar{\psi}_{L} \phi^{\dagger} \psi_{R}\right] .
\end{aligned}
$$

We fixed the mass term in such a way that it is generated by a shift $\phi \rightarrow \phi+m /(2 g)$ in $\phi=(A+i B) / \sqrt{2}$ in the interaction terms; we adopt this procedure in the following. The fermion mass term is then defined by the scalar density formed of a fermion bi-linear (1.18). The fermion propagator is given by

$$
\langle\psi(y) \bar{\psi}(x)\rangle=(-) \frac{a}{H+a m \Gamma_{5}} \gamma_{5}=(-) \frac{a\left(H+a m \Gamma_{5}\right)}{H^{2}+\left(a m \Gamma_{5}\right)^{2}} \gamma_{5} .
$$

Note that $H^{2}$ and $\Gamma_{5}^{2}$ are proportional to a $4 \times 4$ unit Dirac matrix.

The second possible Lagrangian incorporates the continuum chiral symmetry for Yukawa couplings, which is softly broken by the mass term,

$$
\mathcal{L}_{(2)}=\bar{\psi} D \psi+m \bar{\psi} \psi+2 g \bar{\psi}\left(P_{+} \phi P_{+}+P_{-} \phi^{\dagger} P_{-}\right) \psi
$$

but no explicit lattice chiral symmetry even for $m=0$. The propagator is given by

$$
\begin{aligned}
\langle\psi(y) \bar{\psi}(x)\rangle=(-) \frac{a}{H+a m \gamma_{5}} \gamma_{5} & =(-) \frac{a\left(H+a m \gamma_{5}\right)}{H^{2}+a m\left(H \gamma_{5}+\gamma_{5} H\right)+(a m)^{2}} \gamma_{5} \\
& =(-) \frac{a\left(H+a m \gamma_{5}\right)}{H^{2}+2 a m H^{2 k+2}+(a m)^{2}} \gamma_{5}
\end{aligned}
$$


where we used the defining algebra of the Ginsparg-Wilson operator [2. This propagator has a pole structure different from the one naively expected on the basis of the Lagrangian $(2.5)$.

Yet another Lagrangian, which is suggested by the analysis of lattice chiral gauge theory with a real representation of gauge group [27, is

$$
\mathcal{L}_{(3)}=\bar{\psi} D \psi+i m \bar{\psi} \gamma_{5} \psi+2 i g \bar{\psi} \gamma_{5}\left(P_{+} \phi P_{+}+P_{-} \phi^{\dagger} P_{-}\right) \psi
$$

Note that we fix the mass term in such a way that it is generated by a shift $\phi \rightarrow \phi+m /(2 g)$ in the interaction terms. (To be precise, the analysis in [27] fixes the mass term, and we fixed the interaction terms accordingly.) This Lagrangian incorporates a certain aspect of lattice chiral symmetry as is seen by evaluating the propagator

$$
\langle\psi(y) \bar{\psi}(x)\rangle=(-) \frac{a}{H+i a m} \gamma_{5}=(-) \frac{a(H-i a m)}{H^{2}+(a m)^{2}} \gamma_{5}
$$

which has a pole structure naively expected on the basis of the Lagrangian. The factor $i \gamma_{5}$ in the Lagrangian is eliminated by a $\pi / 4$ chiral rotation up to chiral anomaly, which is absent in the present model,

$$
\begin{aligned}
& \psi \rightarrow e^{-i(\pi / 4) \gamma_{5}} \psi, \\
& \bar{\psi} \rightarrow \bar{\psi} e^{-i(\pi / 4) \gamma_{5}}
\end{aligned}
$$

if the kinetic term is invariant under the continuum chiral symmetry. However, the Ginsparg-Wilson operator is not invariant under the continuun chiral symmetry, and thus the factor $\gamma_{5}$ has an intrinsic meaning. In fact we later show that this scheme violates $C P$ symmetry.

\subsection{Majorana reduction and supersymmetry}

We now define a Majorana fermion starting with the Lagrangian for a Dirac fermion. In the analysis of a Majorana fermion, the transpose operation is essential. We employ the convention

$$
\begin{aligned}
& C \gamma^{\mu} C^{-1}=-\left(\gamma^{\mu}\right)^{T}, \\
& C \gamma_{5} C^{-1}=\gamma_{5}^{T}, \\
& C^{\dagger} C=1, \quad C^{T}=-C .
\end{aligned}
$$

We then have

$$
\begin{aligned}
& C^{T}=-C \\
& \left(C \gamma_{5}\right)^{T}=-C \gamma_{5} \\
& (C D)^{T}=-C D, \quad\left(C \gamma_{5} \Gamma_{5}\right)^{T}=-C \gamma_{5} \Gamma_{5} \\
& (C H)^{T}=\left(C \gamma_{5} a D\right)^{T}=-C a D \gamma_{5} \neq-C H, \quad\left(C \Gamma_{5}\right)^{T} \neq-C \Gamma_{5} .
\end{aligned}
$$

\footnotetext{
${ }^{2}$ This propagator approaches for large $k$ to $\sim(-) \frac{a\left(H+a m \gamma_{5}\right)}{H^{2}+(a m)^{2}} \gamma_{5}$, since $H^{2} \leq 1$ and almost all the eigenvalues of $H$ are less than unity.
} 
The basic idea of the Majorana reduction is to decompose a Dirac fermion $\psi$ into a sum of two Majorana fermions $\chi$ and $\eta$, which are Grassmann numbers, as

$$
\begin{aligned}
& \psi=(\chi+i \eta) / \sqrt{2} \\
& \bar{\psi}=\left(\chi^{T} C-i \eta^{T} C\right) / \sqrt{2} .
\end{aligned}
$$

\subsubsection{Majorana fermion with lattice chiral symmetry}

We start with the Dirac fermion with lattice chiral symmetry (2.3) and apply the above substitution (2.14). We naively expect

$$
\begin{aligned}
\mathcal{L}_{(1)} & =\frac{1}{2} \chi^{T} C D \chi+\frac{1}{2} m \chi^{T} C \gamma_{5} \Gamma_{5} \chi+g \chi^{T} C\left(P_{+} \phi \hat{P}_{+}+P_{-} \phi^{\dagger} \hat{P}_{-}\right) \chi \\
& +\frac{1}{2} \eta^{T} C D \eta+\frac{1}{2} m \eta^{T} C \gamma_{5} \Gamma_{5} \eta+g \eta^{T} C\left(P_{+} \phi \hat{P}_{+}+P_{-} \phi^{\dagger} \hat{P}_{-}\right) \eta
\end{aligned}
$$

but this actually fails if one recalls

$$
\left(P_{+} \phi \hat{P}_{+}+P_{-} \phi^{\dagger} \hat{P}_{-}\right)=\frac{1}{\sqrt{2}}\left(A \gamma_{5} \Gamma_{5}+i B \Gamma_{5}\right)
$$

where we used $\phi=(A+i B) / \sqrt{2}$ : In the coupling of $A$ we have $\left(C \gamma_{5} \Gamma_{5}\right)^{T}=-C \gamma_{5} \Gamma_{5}$ but the difference operator appearing in $\Gamma_{5}$ does not commute with $A(x)$, and in the coupling of the pseudscalar $B(x)$ we have $\left(C \Gamma_{5}\right)^{T} \neq-C \Gamma_{5}$.

To cope with this conflict between lattice chiral symmetry and the Majorana condition of Yukawa couplings, we choose to impose the lattice chiral symmetry and analyze the theory defined by the path integral

$$
\begin{aligned}
& \left.Z=\frac{1}{Z_{0}} \int \mathcal{D} \phi \mathcal{D} \phi^{\dagger} \mathcal{D} F \mathcal{D} F^{\dagger} \sqrt{\operatorname{det}\left[D+m \gamma_{5} \Gamma_{5}+2 g\left(P_{+} \phi \hat{P}_{+}+P_{-} \phi^{\dagger} \hat{P}_{-}\right)\right.}\right] \\
& \times \exp \left\{\int-\phi^{\dagger} D^{\dagger} D \phi+F^{\dagger} F+m\left[F \Gamma \phi+(F \Gamma \phi)^{\dagger}\right]+g\left[F \Gamma \phi^{2}+\left(F \Gamma \phi^{2}\right)^{\dagger}\right]\right\}
\end{aligned}
$$

with the normalization factor

$$
\begin{aligned}
Z_{0} \equiv & \left.\int \mathcal{D} \phi \mathcal{D} \phi^{\dagger} \mathcal{D} F \mathcal{D} F^{\dagger} \sqrt{\operatorname{det}\left[D+m \gamma_{5} \Gamma_{5}\right.}\right] \\
& \times \exp \left\{\int-\phi^{\dagger} D^{\dagger} D \phi+F^{\dagger} F+m\left[F \Gamma \phi+(F \Gamma \phi)^{\dagger}\right]\right\}
\end{aligned}
$$

which cancels the divergence coming from the positive definite term $F^{\dagger} F$. The square root in (2.17) does not define the conventional Pfaffianf for a finite lattice spacing $a$ because of Yukawa couplings. We defined the bosonic part of the action in terms of fermionic operators. The operator $D^{\dagger} D=H^{2} / a^{2}$ is proportional to a $4 \times 4$ unit matrix, but we

\footnotetext{
${ }^{3}$ If $(C O)^{T}=-C O$ for a general operator $O$, the cross term vanishes $\eta^{T} C O \chi-\chi^{T} C O \eta=0$ by using the anti-commuting property of $\chi$ and $\eta$.

${ }^{4}$ One may note that there does not exist a perfect Euclidean Majorana fermion to begin with.
} 
adopt a convention to discard the unit matrix when $D^{\dagger} D=D D^{\dagger}$ appears in the bosonic part of the Lagrangian. In the action $(2.17)$ we multiplied the auxiliary field $F$ in the mass and interaction terms by an extra factor $\Gamma$ with

$$
\Gamma \equiv \sqrt{1-H^{4 k+2}}
$$

to maintain the degeneracy between fermion and boson masses. This expression of $\Gamma$ is formally equal to $\sqrt{\Gamma_{5}^{2}}$, but we again adopt the convention that $\Gamma^{2}$, unlike $\Gamma_{5}^{2}$, does not carry a $4 \times 4$ unit Dirac matrix.

In the perturbative treatment, one can reproduce a result equivalent to (2.17) by analyzing a theory defined by a Dirac fermion

$$
\begin{aligned}
\mathcal{L} & =\bar{\psi} D \psi+m \bar{\psi} \gamma_{5} \Gamma_{5} \psi+2 g \bar{\psi}\left(P_{+} \phi \hat{P}_{+}+P_{-} \phi^{\dagger} \hat{P}_{-}\right) \psi \\
& -\phi^{\dagger} D^{\dagger} D \phi+F^{\dagger} F+m\left[F \Gamma \phi+(F \Gamma \phi)^{\dagger}\right]+g\left[F \Gamma \phi^{2}+\left(F \Gamma \phi^{2}\right)^{\dagger}\right]
\end{aligned}
$$

provided that we adopt the following calculational rules: We divide by a factor of 2 whenever we encounter a fermion loop contribution in perturbation theory. The quantum effects on the fermion lines are treated in the ordinary way. The propagators are given by

$$
\begin{aligned}
& \left\langle\phi \phi^{\dagger}\right\rangle=\frac{a^{2}}{H^{2}+(a m \Gamma)^{2}} \\
& \left\langle F F^{\dagger}\right\rangle=(-) \frac{H^{2}}{H^{2}+(a m \Gamma)^{2}} \\
& \langle F \phi\rangle=\left\langle F^{\dagger} \phi^{\dagger}\right\rangle=(-) \frac{a^{2} m \Gamma}{H^{2}+(a m \Gamma)^{2}} \\
& \langle\psi \bar{\psi}\rangle=\frac{-1}{D+m \gamma_{5} \Gamma_{5}}=(-) \frac{a\left(H+a m \Gamma_{5}\right)}{H^{2}+\left(a m \Gamma_{5}\right)^{2}} \gamma_{5}
\end{aligned}
$$

and other propagators vanish. When we have $H^{2}$ in the bosonic propagators, we adopt the convention to discard the unit Dirac matrix in $H^{2}$.

A justification for our choice of the Lagrangian (2.17), in particular for our choice of mass terms, is that the free Lagrangian after eliminating the auxiliary field (written in the original Majorana fermion )

$$
\begin{aligned}
\mathcal{L}_{0} & =\frac{1}{2} \chi^{T} C D \chi+\frac{1}{2} m \chi^{T} C \gamma_{5} \Gamma_{5} \chi \\
& -\frac{1}{2}\left[A D^{\dagger} D A+B D^{\dagger} D B\right]-\frac{1}{2} m^{2}\left[A \Gamma^{2} A+B \Gamma^{2} B\right] \\
& =\frac{1}{2} \chi^{T} C \gamma_{5}\left[\frac{1}{a} H+m \Gamma_{5}\right] \chi \\
& -\frac{1}{2 a^{2}}\left[A H^{2} A+B H^{2} B\right]-\frac{1}{2} m^{2}\left[A \Gamma^{2} A+B \Gamma^{2} B\right]
\end{aligned}
$$

is invariant under a "SUSY-like transformation"

$$
\begin{aligned}
\delta \chi & =\left(\frac{1}{a} H+m \Gamma_{5}\right) \gamma_{5}\left(A-i \gamma_{5} B\right) \epsilon \\
\delta A & =\epsilon^{T} C \chi \\
\delta B & =-i \epsilon^{T} C \gamma_{5} \chi
\end{aligned}
$$


with a Majorana parameter $\epsilon$. The cancellation of fermionic and bosonic determinants for the free Lagrangian is also confirmed by noting

$$
\sqrt{\operatorname{det}\left[H / a+m \Gamma_{5}\right]}=\left\{\operatorname{det}\left[H / a+m \Gamma_{5}\right]^{2}\right\}^{1 / 4}=\left\{\operatorname{det}\left[H^{2} / a^{2}+m^{2} \Gamma_{5}^{2}\right]\right\}^{1 / 4}
$$

and the fact that $H^{2}$ and $\Gamma_{5}^{2}$ are proportional to a $4 \times 4$ unit matrix. But the symmetry of the full action is not maintained. See Refs. 14] [15] for discussions of the related issue.

In passing, we note that the fermion propagator in a Majorana notation is given by

$$
\left\langle\chi(y) \chi^{T}(x) C\right\rangle=(-) \frac{a}{H+a m \Gamma_{5}} \gamma_{5}
$$

\subsubsection{Majorana fermion with continuum chiral symmetry for Yukawa cou- plings (1)}

We next examine the Lagrangian (2.5) with naive chiral symmetry for mass and Yukawa terms but no lattice chiral symmetry . After the Majorana reduction, one has

$$
\begin{aligned}
\mathcal{L}_{M(2)}= & \frac{1}{2} \chi^{T} C D \chi+\frac{1}{2} m \chi^{T} C \chi+g \chi^{T} C\left(P_{+} \phi P_{+}+P_{-} \phi^{\dagger} P_{-}\right) \chi \\
- & \phi^{\dagger} D_{1}^{\dagger} D_{1} \phi+F^{\dagger} F+F\left(m+D_{2}\right) \phi+\left(F\left(m+D_{2}\right) \phi\right)^{\dagger} \\
& +g\left[F \phi^{2}+\left(F \phi^{2}\right)^{\dagger}\right] \\
= & \frac{1}{2} \chi^{T} C D_{1} \chi+\frac{1}{2} m \chi^{T} C \chi+g \chi^{T} C\left(P_{+} \phi P_{+}+P_{-} \phi^{\dagger} P_{-}\right) \chi \\
- & \phi^{\dagger} D_{1}^{\dagger} D_{1} \phi+F^{\dagger} F+m F \phi+(m F \phi)^{\dagger}+g\left[F \phi^{2}+\left(F \phi^{2}\right)^{\dagger}\right] \\
& +\frac{1}{2} \chi^{T} C D_{2} \chi+F D_{2} \phi+\left(F D_{2} \phi\right)^{\dagger} .
\end{aligned}
$$

The Majorana reduction works naturally, but we separated the kinetic term of the fermion into two parts

$$
\begin{aligned}
& D_{1} \equiv \frac{1}{2}\left(D-D^{\dagger}\right)=\frac{1}{2 a}\left(\gamma_{5} H-H \gamma_{5}\right) \propto \not p \\
& D_{2} \equiv \frac{1}{2}\left(D+D^{\dagger}\right)=\frac{1}{2 a}\left(\gamma_{5} H+H \gamma_{5}\right)=\frac{1}{a} H^{2 k+2}
\end{aligned}
$$

to ensure the degeneracy of fermion and boson mass spectrum and also the one-loop tadpole cancellation. Here we used the fact that $D_{2}$ is independent of Dirac matrices for the free fermion case. Note that in general

$$
\begin{aligned}
& \left\{\gamma_{5}, D_{1}\right\}=\frac{1}{2 a}\left\{\gamma_{5},\left(\gamma_{5} H-H \gamma_{5}\right)\right\}=0, \\
& {\left[\gamma_{5}, D_{2}\right]=\frac{1}{2 a}\left[\gamma_{5},\left(\gamma_{5} H+H \gamma_{5}\right)\right]=0 .}
\end{aligned}
$$

In the Lagrangian (2.28), the kinetic (Kähler) term is given by

$$
\mathcal{L}_{M(2)}{ }_{k i n}=\frac{1}{2} \chi^{T} C D_{1} \chi-\phi^{\dagger} D_{1}^{\dagger} D_{1} \phi+F^{\dagger} F
$$


and a part of the Ginsparg-Wilson operator is transferred to a potential part (which however contains derivative operators)

$$
\mathcal{L}_{M(2) \text { pot }}=\frac{1}{2} \chi^{T} C D_{2} \chi+F D_{2} \phi+\left(F D_{2} \phi\right)^{\dagger}
$$

which is treated in a manner analogous to the Wilson term in the conventional lattice formulation. This potential term induces a hard breaking of continuum chiral symmetry.

The propagators are given by

$$
\begin{aligned}
\left\langle\phi \phi^{\dagger}\right\rangle & =\frac{1}{D_{1}^{\dagger} D_{1}+\left(m+D_{2}\right)^{2}} \\
& =\frac{a^{2}}{H^{2}+m a\left(\gamma_{5} H+H \gamma_{5}\right)+(a m)^{2}} \\
& =\frac{a^{2}}{H^{2}+2 m a H^{2 k+2}+(a m)^{2}}, \\
\left\langle F F^{\dagger}\right\rangle & =(-) \frac{a^{2} D_{1}^{\dagger} D_{1}}{H^{2}+2 m a H^{2 k+2}+(a m)^{2}} \\
\langle F \phi\rangle & =\left\langle F^{\dagger} \phi^{\dagger}\right\rangle=(-) \frac{H^{2}-H^{4 k+4}}{H^{2}+2 m a H^{2 k+2}+(a m)^{2}} \\
& =(-) \frac{a\left(a m+H^{2 k+2}\right)}{H^{2}+2 m a H^{2 k+2}+(a m)^{2}}, \\
\left\langle\chi \chi^{T} C\right\rangle & =\frac{-1}{D+m}=(-) \frac{a}{H^{2}+a m \gamma_{5}} \gamma_{5} \\
& =(-) \frac{a\left(H+a m \gamma_{5}\right)}{H^{2}+2 m a H^{2 k+2}+(a m)^{2}} \gamma_{5}
\end{aligned}
$$

and other propagators vanish.

This scheme corresponds to the analysis of Bartels and Kramer [17] on the basis of the conventional Wilson fermion, if one identifies $D_{1}$ with the naive lattice Dirac operator and $D_{2}$ as the Wilson term. This decomposition crucially depends on the fact that $D_{2}$ is independent of Dirac matrices, which is not the case in the presence of background gauge field, for example. (In such a case, one need to define $D_{2}$ in the bosonic sector independently from $D_{2}$ in the fermionic sector.) In this sense, the Ginsparg-Wilson operator is less flexible than the naive lattice fermion with a Wilson term[17] when applied to a scalar multiplet. 


\subsubsection{Majorana fermion with continuum chiral symmetry for Yukawa cou- plings (2)}

We next examine the Lagrangian (2.7) with naive chiral symmetry for mass and Yukawa terms but with an extra factor of $\gamma_{5}$. After the Majorana reduction, one has

$$
\begin{aligned}
\mathcal{L}_{M(3)} & =\frac{1}{2} \chi^{T} C D \chi+\frac{i}{2} m \chi^{T} C \gamma_{5} \chi+i g \chi^{T} C \gamma_{5}\left(P_{+} \phi P_{+}+P_{-} \phi^{\dagger} P_{-}\right) \chi \\
& -\phi^{\dagger} D^{\dagger} D \phi+F^{\dagger} F+m F \phi+m(F \phi)^{\dagger}+g\left[F \phi^{2}+\left(F \phi^{2}\right)^{\dagger}\right] .
\end{aligned}
$$

The Majorana reduction works naturally, and the propagators are given by

$$
\begin{aligned}
\left\langle\phi \phi^{\dagger}\right\rangle & =\frac{1}{D^{\dagger} D+m^{2}} \\
& =\frac{a^{2}}{H^{2}+(a m)^{2}} \\
\left\langle F F^{\dagger}\right\rangle & =(-) \frac{H^{2}}{H^{2}+(a m)^{2}} \\
\langle F \phi\rangle & =\left\langle F^{\dagger} \phi^{\dagger}\right\rangle=(-) \frac{a^{2} m}{H^{2}+(a m)^{2}} \\
\left\langle\chi \chi^{T} C\right\rangle & =\frac{-1}{D+i m \gamma_{5}}=(-) \frac{a}{H+i a m} \gamma_{5} \\
& =(-) \frac{a(H-i a m)}{H^{2}+(a m)^{2}} \gamma_{5}
\end{aligned}
$$

and other propagators vanish.

This scheme is the simplest in many respects, but this scheme leads to non-vanishing fermion tadpole contributions for both of the scalar $A$ and the pseudscalar $B$. This

means that the vacuum breaks $\mathrm{CP}$ symmetry. To be precise, the one-loop fermion tadpole diagram is evaluated by

$$
\begin{aligned}
& \frac{1}{2} \operatorname{Tr} \frac{1}{D+i m \gamma_{5}} 2 i g \gamma_{5}\left(P_{+} \phi P_{+}+P_{-} \phi^{\dagger} P_{-}\right) \\
& =\frac{2 i g a}{2} \operatorname{Tr}\left(P_{+} \phi+P_{-} \phi^{\dagger}\right) \frac{H-i m a}{H^{2}+(m a)^{2}} \\
& =\frac{2 i g a}{4} \operatorname{Tr}\left[\left(\phi+\phi^{\dagger}\right)+\left(\phi-\phi^{\dagger}\right) \gamma_{5}\right] \frac{H-i m a}{H^{2}+(m a)^{2}} \\
& =\frac{2 g a}{4}\left(\phi+\phi^{\dagger}\right) \operatorname{Tr} \frac{i H+m a}{H^{2}+(m a)^{2}} \\
& +\frac{2 i g a}{4}\left(\phi-\phi^{\dagger}\right) \operatorname{Tr} \frac{\gamma_{5} H}{H^{2}+(m a)^{2}} \\
& =\frac{\sqrt{2} g m a^{2}}{2} A \operatorname{Tr} \frac{1}{H^{2}+(m a)^{2}} \\
& -\frac{\sqrt{2} g a}{2} B \operatorname{Tr} \frac{H^{2 k+2}}{H^{2}+(m a)^{2}}
\end{aligned}
$$




$$
\begin{aligned}
& =\frac{\sqrt{2} g m}{2 a^{2}} \operatorname{Atr} \int_{-\pi}^{\pi} \frac{d^{4} k}{(2 \pi)^{4}} \frac{1}{H^{2}(k)+(m a)^{2}} \\
& -\frac{\sqrt{2} g}{2 a^{3}} \operatorname{Btr} \int_{-\pi}^{\pi} \frac{d^{4} k}{(2 \pi)^{4}} \frac{H^{2 k+2}(k)}{H^{2}(k)+(m a)^{2}}
\end{aligned}
$$

where we used the fact that $H^{2}$ is independent of Dirac matrices and $\gamma_{5} H+H \gamma_{5}=2 H^{2 k+2}$. We also rescaled the loop momentum $a k_{\mu} \rightarrow k_{\mu}$, and the remaining trace is over Dirac indices. The tadpole for the scalar $A$ is quadratically divergent and it is cancelled by a scalar one-loop tadpole contribution. On the other hand, the tadpole for the pseudscalar $B$ gives cubic divergence, and it cannot be cancelled by scalar loop diagrams unless one introduces complicated CP violating interactions among $\phi$ and $F$.

In passing we note that our scheme, which uses the scalar density (1.18) as a mass term, provides a viable alternative to a Majorana representation of a (massive) chiral gauge theory with a real representation of gauge group discussed in 27], namely

$$
\mathcal{L}=\frac{1}{2} \chi^{T} C D \chi+\frac{1}{2} m \chi^{T} C \gamma_{5} \Gamma_{5} \chi
$$

if one includes gauge field in $D$ and adds a Lagrangian for the gauge field. The fermion propagator in this case is given by $(2.27)$.

\section{Weyl Representation and Holomorphic Properties}

We have so far examined the lattice Majorana representation. We now discuss the lattice Weyl representation, which is more fundamental from a view point of supersymmetryf. An analysis of weyl fermion with a real representation of gauge group suggests the following Lagrangian 27]

$$
\mathcal{L}_{(4)}^{(0)}=\bar{\psi}_{L} D \psi_{L}+\frac{1}{2} i m \psi_{L}^{T} B \psi_{L}-\frac{1}{2} i m \bar{\psi}_{L} B^{-1} \bar{\psi}_{L}^{T}
$$

Here $B=C \gamma_{5}$, and this expression is consistent if one recalls $\psi_{L}=\hat{P}_{-} \psi, \bar{\psi}_{L}=\bar{\psi} P_{+}$and the properties

$$
\begin{aligned}
B \hat{P}_{-} & =C \gamma_{5} \frac{1}{2}\left(1-\hat{\gamma}_{5}\right)=C \gamma_{5} \frac{1}{2}\left(1+\gamma_{5}-2 \Gamma_{5}\right) \\
& =-\left(C \gamma_{5} \frac{1}{2}\left(1+\gamma_{5}-2 \Gamma_{5}\right)\right)^{T}=-\hat{P}_{-}^{T} B^{T}=\hat{P}_{-}^{T} B, \\
P_{+} B^{-1} & =B^{-1} B P_{+} B^{-1}=B^{-1} P_{+}^{T}
\end{aligned}
$$

where we used $B^{T}=-B$. Note that if one replaces $B$ by $C$ in (3.1), it does not work since the first relation in (3.2) does not hold; $C \Gamma_{5} \neq-\left(C \Gamma_{5}\right)^{T}$. We adopt the Lagrangian (3.1) as a basis of our analysis since we have a free Dirac operator $D$.

\footnotetext{
${ }^{5}$ In continuum (and Minkowski) theory the equivalence of the Majorana and Weyl representations is trivially valid, but on the lattice their equivalence is not evident.
} 
A natural way to introduce the Yukawa coupling is to consider

$$
\begin{aligned}
\mathcal{L}_{L(4)}= & \bar{\psi}_{L} D \psi_{L}+\frac{1}{2} i m \psi_{L}^{T} B \psi_{L}-\frac{1}{2} i m \bar{\psi}_{L} B^{-1} \bar{\psi}_{L}^{T} \\
& +i g \psi_{L}^{T} B \phi \psi_{L}-i g \bar{\psi}_{L} B^{-1} \phi^{\dagger} \bar{\psi}_{L}^{T} \\
& -\phi^{\dagger} D^{\dagger} D \phi+F^{\dagger} F+m F \phi+m(F \phi)^{\dagger}+g\left[F \phi^{2}+\left(F \phi^{2}\right)^{\dagger}\right]
\end{aligned}
$$

which is satisfactory from a view point of holomorphic properties in the sense that the potential terms consist of either $\left(\phi, \psi_{L}, F\right)$ or $\left(\phi^{\dagger}, \bar{\psi}_{L}, F^{\dagger}\right)$, and these sets of fields mix only in the kinetic terms

$$
\begin{aligned}
\mathcal{L}_{L(4) \text { kin }} & =\bar{\psi}_{L} D \psi_{L}-\phi^{\dagger} D^{\dagger} D \phi+F^{\dagger} F \\
& =\bar{\psi}_{L} \frac{H}{a} \psi_{L}-\phi^{\dagger}\left(\frac{H}{a}\right)^{2} \phi+F^{\dagger} F .
\end{aligned}
$$

The mass terms are also generated by a shift $\phi \rightarrow \phi+m /(2 g)$ in the interaction terms.

However the Yukawa coupling in this formula (3.3) is not consistent as a Weyl decomposition. This fact is seen as follows: One may first define a Lagrangian in terms of unconstrained variables $\psi$ and $\bar{\psi}$ as

$$
\begin{aligned}
\mathcal{L}_{(4)}= & \bar{\psi} D \psi+\frac{1}{2} i m \psi^{T} B \psi-\frac{1}{2} i m \bar{\psi} B^{-1} \bar{\psi}^{T} \\
& +i g \psi^{T} B \phi \psi-i g \bar{\psi} \phi^{\dagger} B^{-1} \bar{\psi}^{T} \\
& -\phi^{\dagger} D^{\dagger} D \phi+F^{\dagger} F+m F \phi+m(F \phi)^{\dagger}+g\left[F \phi^{2}+\left(F \phi^{2}\right)^{\dagger}\right]
\end{aligned}
$$

and define the path integral

$$
\int \mathcal{D} \psi \mathcal{D} \bar{\psi} \exp \left[\int \mathcal{L}_{(4)}\right]
$$

A Weyl decomposition means to rewrite this path integral as a product of left and right components as ( for the moment, by paying attention only to the fermion sector)

$$
\int \mathcal{D} \psi \mathcal{D} \bar{\psi} \exp \left[\int \mathcal{L}_{(4)}\right]=\int \mathcal{D} \psi_{L} \mathcal{D} \bar{\psi}_{L} \exp \left[\int \mathcal{L}_{L(4)}\right] \int \mathcal{D} \psi_{R} \mathcal{D} \bar{\psi}_{R} \exp \left[\int \mathcal{L}_{R(4)}\right] .
$$

But this fails since we have a cross term in the Yukawa coupling

$$
\begin{aligned}
& i g \int\left[\psi_{L}^{T} B \phi \psi_{R}+\psi_{R}^{T} B \phi \psi_{L}\right] \\
& =2 i g \int \psi_{R}^{T} B \phi \psi_{L}=2 i g \int \psi_{R}^{T} B \phi \hat{P}_{-} \psi \\
& =2 i g \int \psi_{R}^{T} \phi \hat{P}_{-}^{T} B \psi=2 i g \int \psi_{R}^{T}\left[\phi, \hat{P}_{-}^{T}\right] B \psi \\
& =2 i g \int \psi_{R}^{T}\left[\phi,\left(H^{2 k+1}\right)^{T}\right] B \psi \neq 0
\end{aligned}
$$

where we used $B^{T}=-B, \psi_{R}^{T} \hat{P}_{-}^{T}=0$ and an explicit expression of $\hat{P}_{-}^{T}$. For the unconstrained variable $\psi$, the vanishing condition of this cross term requires

$$
\psi_{R}^{T}\left[\phi(x),\left(H^{2 k+1}\right)^{T}\right]=0
$$


which however does not hold , since the difference operator appearing in $H$ does not commute with $\phi(x)$.

In view of the absence of the precise Weyl decomposition in the present framework, one may use the Lagrangian (3.5) and divide all the fermion loop diagrams by a factor of 2 in perturbative calculations. The propagators in (3.5) are given by

$$
\begin{aligned}
\langle\psi \bar{\psi}\rangle & =\frac{-1}{D^{\dagger} D+m^{2}} D^{\dagger}=\frac{-a}{H^{2}+(a m)^{2}} H \gamma_{5} \\
\left\langle\psi \psi^{T} B\right\rangle & =\frac{i m}{D^{\dagger} D+m^{2}}=\frac{i m a^{2}}{H^{2}+(a m)^{2}} \\
\left\langle B^{-1} \bar{\psi}^{T} \bar{\psi}\right\rangle & =\frac{-i m}{D^{\dagger} D+m^{2}}=\frac{-i m a^{2}}{H^{2}+(a m)^{2}} \\
\left\langle\phi \phi^{\dagger}\right\rangle & =\frac{1}{D^{\dagger} D+m^{2}}=\frac{a^{2}}{H^{2}+(a m)^{2}} \\
\left\langle F F^{\dagger}\right\rangle & =(-) \frac{H^{2}}{H^{2}+(a m)^{2}} \\
\langle F \phi\rangle & =\left\langle F^{\dagger} \phi^{\dagger}\right\rangle=(-) \frac{a^{2} m}{H^{2}+(a m)^{2}} .
\end{aligned}
$$

For the Lagrangian (3.5), one can confirm that no $\mathrm{CP}$ violation appears in one-loop level fermion tadpoles and that the fermion tadpoles are precisely cancelled by scalar tadpoles. The Lagrangian (3.5) preserves a certain aspect of holomorphic properties but the information of lattice chiral symmetry is lost in Yukawa couplings t; consequently, the potential part including fermion mass terms retains a good renormalization property but the fermion self-energy part induces a linear divergence (and thus a Dirac mass ). Incidentally, if the chiral decomposition (3.7) should be exact, no linear divergence would appear in the one-loop fermion self-energy for (3.5).

We thus recognize a conflict between the lattice chiral symmetry and Yukawa couplings for the Weyl representation also. An alternative Weyl representation is obtained by rewriting $\mathcal{L}_{M(2)}$ in (2.28) in terms of two-component spinors. In this sense, only the Lagrangian (2.28) is consistent with the Majorana or Weyl condition, but of course (2.28) explicitly breaks lattice chiral symmetry by Yukawa couplings from the beginning; besides the term $\mathcal{L}_{M(2) \text { pot }}$ in (2.32) breaks $U(1)$ in $U(1) \times U(1)_{R}$ symmetry in the kinetic (Kähler) term $\mathcal{L}_{M(2)_{k i n}}(2.31)$ even for $m=g=0$; here $U(1)_{R}$ stands for R-symmetry.

\section{One-loop Perturbative Analysis}

In this Section we examine in some detail the lattice regularization of the Wess-Zumino model (2.17) which preserves lattice chiral symmetry. We show that the corrections to all

\footnotetext{
${ }^{6}$ Alternative way to see this complication is to note that the interaction term $i g \int \psi_{L}^{T} B \phi \psi_{L}$ in $(3.3)$ does not satisfy the consistency condition in the sense of (3.2) because of the presence of $\phi(x)$. The field $\phi(x)$ connects $\psi_{L}$ to $\psi_{R}^{T}$ as in $(3.8)$.

${ }^{7}$ This property is somewhat complementary to $(2.20)$, where lattice chiral symmetry is manifest but holomorphic properties are not obvious.
} 
the interaction terms (including mass terms ) of all the field variables $\psi$ and $\phi$ vanish in the one-loop level for a finite lattice spacing $a$ when renormalized at vanishing momenta. This in particular shows that the quadratic divergences in the mass term of the scalar particle are exactly cancelled in the one-loop level. In this sense, our regularization preserves a certain essential aspect of the supersymmetric model.

\subsection{Fermionic One-loop Contributions}

The one-loop fermion contribution is evaluated by

$$
\begin{aligned}
& \sqrt{\operatorname{det}\left[D+m \gamma_{5} \Gamma_{5}+2 g\left(P_{+} \phi \hat{P}_{+}+P_{-} \phi^{\dagger} \hat{P}_{-}\right)\right]} \\
& =\exp \left\{\frac{1}{2} \operatorname{Tr} \ln \left[D+m \gamma_{5} \Gamma_{5}+2 g\left(P_{+} \phi \hat{P}_{+}+P_{-} \phi^{\dagger} \hat{P}_{-}\right)\right]\right\} \\
& =\exp \left\{\frac{1}{2} \operatorname{Tr} \ln \left[D+m \gamma_{5} \Gamma_{5}\right]+\frac{1}{2} \operatorname{Tr} \frac{1}{D+m \gamma_{5} \Gamma_{5}} 2 g\left(P_{+} \phi \hat{P}_{+}+P_{-} \phi^{\dagger} \hat{P}_{-}\right)\right. \\
& -\frac{1}{4} \operatorname{Tr} \frac{1}{D+m \gamma_{5} \Gamma_{5}} 2 g\left(P_{+} \phi \hat{P}_{+}+P_{-} \phi^{\dagger} \hat{P}_{-}\right) \frac{1}{D+m \gamma_{5} \Gamma_{5}} 2 g\left(P_{+} \phi \hat{P}_{+}+P_{-} \phi^{\dagger} \hat{P}_{-}\right) \\
& +\ldots\}
\end{aligned}
$$

\subsubsection{Tadpole Diagrams}

We first examine the tadpole terms, namely, terms linear in $\phi$ and $\phi^{\dagger}$. We have

$$
\begin{aligned}
& \frac{1}{2} \operatorname{Tr} \frac{1}{D+m \gamma_{5} \Gamma_{5}} 2 g\left(P_{+} \phi \hat{P}_{+}+P_{-} \phi^{\dagger} \hat{P}_{-}\right) \\
& =\frac{2 g a}{2} \operatorname{Tr}\left(P_{+} \phi \Gamma_{5}+P_{-} \phi^{\dagger} \Gamma_{5}\right) \frac{H+m a \Gamma_{5}}{H^{2}+\left(m a \Gamma_{5}\right)^{2}} \\
& =\frac{2 g m a^{2}}{2} \operatorname{Tr}\left(P_{+} \phi+P_{-} \phi^{\dagger}\right) \frac{\Gamma_{5}^{2}}{H^{2}+\left(m a \Gamma_{5}\right)^{2}} \\
& =\frac{2 g m a^{2}}{4}\left(\phi+\phi^{\dagger}\right) \operatorname{Tr} \frac{\Gamma_{5}^{2}}{H^{2}+\left(m a \Gamma_{5}\right)^{2}}
\end{aligned}
$$

where we used $P_{ \pm} \Gamma_{5} H P_{ \pm}=0$ and the fact that $H^{2}$ and $\Gamma_{5}^{2}$ are independent of $\gamma^{\mu}$ matrices. The trace for fermion loop amplitudes includes the integral over internal momentum as well as the sum over Dirac indices, and the above integral is quadratically divergent?. We also set the momenta carried by external fields at 0 . Note that only the scalar component $\phi+\phi^{\dagger}=\sqrt{2} A$ develops the vacuum value. The trace over Dirac matrices gives an extra factor of 4 when compared to the scalar contribution.

\footnotetext{
${ }^{8}$ The power of divergence in the limit $a \rightarrow 0$ is read from the powers of $a$ appearing in front of these expressions. For example, $a^{2}$ corresponds to quadratic divergence and $a^{4}$ corresponds to logarithmic divergence.
} 


\subsubsection{Self-energy Correction for Scalar Particles}

The self-energy corrections of scalar particles by fermion loop diagrams are given by

$$
\begin{aligned}
& -\frac{1}{4} \operatorname{Tr} \frac{1}{D+m \gamma_{5} \Gamma_{5}} 2 g\left(P_{+} \phi \hat{P}_{+}+P_{-} \phi^{\dagger} \hat{P}_{-}\right) \frac{1}{D+m \gamma_{5} \Gamma_{5}} 2 g\left(P_{+} \phi \hat{P}_{+}+P_{-} \phi^{\dagger} \hat{P}_{-}\right) \\
& =-\frac{(2 g a)^{2}}{4} \operatorname{Tr} \frac{H+m a \Gamma_{5}}{H^{2}+\left(m a \Gamma_{5}\right)^{2}}\left(P_{+} \phi \Gamma_{5}+P_{-} \phi^{\dagger} \Gamma_{5}\right) \frac{H+m a \Gamma_{5}}{H^{2}+\left(m a \Gamma_{5}\right)^{2}}\left(P_{+} \phi \Gamma_{5}+P_{-} \phi^{\dagger} \Gamma_{5}\right) \\
& =-\frac{(2 g a)^{2}}{4} \\
& \times\left[2 \operatorname{Tr} P_{-} \phi^{\dagger} \frac{\Gamma_{5} H}{H^{2}+\left(m a \Gamma_{5}\right)^{2}} P_{+} \phi \frac{\Gamma_{5} H}{H^{2}+\left(m a \Gamma_{5}\right)^{2}}\right. \\
& +\operatorname{Tr} P_{+} \phi \frac{m a \Gamma_{5}^{2}}{H^{2}+\left(m a \Gamma_{5}\right)^{2}} P_{+} \phi \frac{m a \Gamma_{5}^{2}}{H^{2}+\left(m a \Gamma_{5}\right)^{2}} \\
& \left.+\operatorname{Tr} P_{-} \phi^{\dagger} \frac{m a \Gamma_{5}^{2}}{H^{2}+\left(m a \Gamma_{5}\right)^{2}} P_{-} \phi^{\dagger} \frac{m a \Gamma_{5}^{2}}{H^{2}+\left(m a \Gamma_{5}\right)^{2}}\right] \\
& =-\frac{(2 g a)^{2}}{4} \\
& \times\left[-\operatorname{Tr} \phi^{\dagger} \frac{H \Gamma_{5}}{H^{2}+\left(m a \Gamma_{5}\right)^{2}} \phi \frac{\Gamma_{5} H}{H^{2}+\left(m a \Gamma_{5}\right)^{2}}\right. \\
& +\frac{(m a)^{2}}{2} \operatorname{Tr} \phi \frac{\Gamma_{5}^{2}}{H^{2}+\left(m a \Gamma_{5}\right)^{2}} \phi \frac{\Gamma_{5}^{2}}{H^{2}+\left(m a \Gamma_{5}\right)^{2}} \\
& \left.+\frac{(m a)^{2}}{2} \operatorname{Tr} \phi^{\dagger} \frac{\Gamma_{5}^{2}}{H^{2}+\left(m a \Gamma_{5}\right)^{2}} \phi^{\dagger} \frac{\Gamma_{5}^{2}}{H^{2}+\left(m a \Gamma_{5}\right)^{2}}\right]
\end{aligned}
$$

where we used $P_{ \pm} \Gamma_{5} H=-H \Gamma_{5} P_{\mp}, P_{ \pm} \Gamma_{5} H P_{ \pm}=0$ and $P_{ \pm} \Gamma_{5}^{2} P_{\mp}=0$. The first term is quadratically divergent, and the last two terms are logarithmically divergent. When one sets the external momentum at 0 to define the mass renormalization factor, these expressions are written as

$$
\begin{aligned}
& \frac{(2 g a)^{2}}{4} \phi^{\dagger} \phi \operatorname{Tr}\left[\frac{\Gamma_{5}^{2}}{H^{2}+\left(m a \Gamma_{5}\right)^{2}}\right] \\
& -\frac{(2 g a)^{2}(m a)^{2}}{8}\left(2 \phi^{\dagger} \phi+\phi^{\dagger} \phi^{\dagger}+\phi \phi\right) \operatorname{Tr}\left[\frac{\Gamma_{5}^{2}}{H^{2}+\left(m a \Gamma_{5}\right)^{2}} \frac{\Gamma_{5}^{2}}{H^{2}+\left(m a \Gamma_{5}\right)^{2}}\right]
\end{aligned}
$$

where the first term gives subtractive renormalization and the remaining 3 terms give multiplicative renormalization in the conventional classification. But these terms do not appear in the bare Lagrangian with the auxiliarly field $F(2.17)$ and (2.20), and they should be precisely cancelled by scalar contributions. To discuss the cancellation of these

terms by scalar loop contributions, we need to take account of an extra factor of 4 arising from the trace over Dirac matrices. 


\subsubsection{Triple Couplings of Scalars}

The fermion contribution to triple couplings is given by

$$
\begin{aligned}
& \frac{1}{6} \operatorname{Tr}\left\{\frac{a}{H+m a \Gamma_{5}} \gamma_{5} 2 g\left[P_{+} \phi \hat{P}_{+}+P_{-} \phi^{\dagger} \hat{P}_{-}\right]\right. \\
& \times \frac{a}{H+m a \Gamma_{5}} \gamma_{5} 2 g\left[P_{+} \phi \hat{P}_{+}+P_{-} \phi^{\dagger} \hat{P}_{-}\right] \\
& \left.\times \frac{a}{H+m a \Gamma_{5}} \gamma_{5} 2 g\left[P_{+} \phi \hat{P}_{+}+P_{-} \phi^{\dagger} \hat{P}_{-}\right]\right\} \\
& =\frac{4(g a)^{3}}{3} \operatorname{Tr}\left\{\frac{H+m a \Gamma_{5}}{H^{2}+\left(m a \Gamma_{5}\right)^{2}}\left[P_{+} \phi \Gamma_{5}+P_{-} \phi^{\dagger} \Gamma_{5}\right]\right. \\
& \times \frac{H+m a \Gamma_{5}}{H^{2}+\left(m a \Gamma_{5}\right)^{2}}\left[P_{+} \phi \Gamma_{5}+P_{-} \phi^{\dagger} \Gamma_{5}\right] \\
& \left.\times \frac{H+m a \Gamma_{5}}{H^{2}+\left(m a \Gamma_{5}\right)^{2}}\left[P_{+} \phi \Gamma_{5}+P_{-} \phi^{\dagger} \Gamma_{5}\right]\right\} .
\end{aligned}
$$

By using the relations $P_{ \pm} \Gamma_{5} H=-H \Gamma_{5} P_{\mp}$ and $P_{ \pm} \Gamma_{5} H P_{ \pm}=0$, we have, for example,

$$
\begin{aligned}
& \frac{4(g a)^{3}}{3} \operatorname{Tr}\left\{\phi P_{+} \Gamma_{5} \frac{H+m a \Gamma_{5}}{H^{2}+\left(m a \Gamma_{5}\right)^{2}} \phi P_{+} \Gamma_{5} \frac{H+m a \Gamma_{5}}{H^{2}+\left(m a \Gamma_{5}\right)^{2}}\right. \\
& \left.\times \phi P_{+} \Gamma_{5} \frac{H+m a \Gamma_{5}}{H^{2}+\left(m a \Gamma_{5}\right)^{2}}\right\} \\
& =\frac{4(g a)^{3}(m a)^{3}}{3} \\
& \times \operatorname{Tr}\left\{\phi P_{+} \frac{\Gamma_{5}^{2}}{H^{2}+\left(m a \Gamma_{5}\right)^{2}} \phi P_{+} \frac{\Gamma_{5}^{2}}{H^{2}+\left(m a \Gamma_{5}\right)^{2}} \phi P_{+} \frac{\Gamma_{5}^{2}}{H^{2}+\left(m a \Gamma_{5}\right)^{2}}\right\}
\end{aligned}
$$

which is convergent. Similarly, we can confirm that the coupling $\phi^{\dagger} \phi^{\dagger} \phi^{\dagger}$ is convergent.

We next examine

$$
\begin{aligned}
& \frac{4(g a)^{3}}{3} \operatorname{Tr}\left\{\phi^{\dagger} P_{-} \Gamma_{5} \frac{H+m a \Gamma_{5}}{H^{2}+\left(m a \Gamma_{5}\right)^{2}} \phi P_{+} \Gamma_{5} \frac{H+m a \Gamma_{5}}{H^{2}+\left(m a \Gamma_{5}\right)^{2}}\right. \\
& \left.\times \phi P_{+} \Gamma_{5} \frac{H+m a \Gamma_{5}}{H^{2}+\left(m a \Gamma_{5}\right)^{2}}\right\} \\
& =\frac{4(g a)^{3} m a}{3} \\
& \times \operatorname{Tr}\left\{\phi^{\dagger} P_{-} \frac{\Gamma_{5} H}{H^{2}+\left(m a \Gamma_{5}\right)^{2}} \phi P_{+} \frac{\Gamma_{5}^{2}}{H^{2}+\left(m a \Gamma_{5}\right)^{2}} \phi P_{+} \frac{\Gamma_{5} H}{H^{2}+\left(m a \Gamma_{5}\right)^{2}}\right\}
\end{aligned}
$$

which is logarithmically divergent. We thus set the external momenta at 0 to define the renormalization factor at vanishing momenta, and we obtain

$$
-\frac{4(g a)^{3} m a}{3} \phi^{\dagger} \phi \phi
$$




$$
\begin{aligned}
& \times \operatorname{Tr}\left\{\frac{H \Gamma_{5}}{H^{2}+\left(m a \Gamma_{5}\right)^{2}} P_{+} \frac{\Gamma_{5}^{2}}{H^{2}+\left(m a \Gamma_{5}\right)^{2}} \frac{\Gamma_{5} H}{H^{2}+\left(m a \Gamma_{5}\right)^{2}}\right\} \\
& =-\frac{4(g a)^{3} m a}{6} \phi^{\dagger} \phi \phi \\
& \times \operatorname{Tr}\left\{\frac{H^{2}}{H^{2}+\left(m a \Gamma_{5}\right)^{2}} \frac{\Gamma_{5}^{2}}{H^{2}+\left(m a \Gamma_{5}\right)^{2}} \frac{\Gamma_{5}^{2}}{H^{2}+\left(m a \Gamma_{5}\right)^{2}}\right\} .
\end{aligned}
$$

Since we have 3 combinations for this choice, we have the numerical coefficient

$$
-\frac{4(g a)^{3} m a}{6} \times 3 \times 4=-\frac{48(g a)^{3} m a}{6}
$$

where the last factor of 4 comes from the trace over Dirac matrices.

We have the same numerical coefficient for the combination $\phi^{\dagger} \phi^{\dagger} \phi$.

\subsubsection{Quartic Couplings}

The quartic couplings from the fermion contribution are given by

$$
\begin{aligned}
& -\frac{(2 g a)^{4}}{8} \operatorname{Tr}\left\{\frac{H+m a \Gamma_{5}}{H^{2}+\left(m a \Gamma_{5}\right)^{2}}\left[P_{+} \phi \Gamma_{5}+P_{-} \phi^{\dagger} \Gamma_{5}\right]\right. \\
& \times \frac{H+m a \Gamma_{5}}{H^{2}+\left(m a \Gamma_{5}\right)^{2}}\left[P_{+} \phi \Gamma_{5}+P_{-} \phi^{\dagger} \Gamma_{5}\right] \\
& \times \frac{H+m a \Gamma_{5}}{H^{2}+\left(m a \Gamma_{5}\right)^{2}}\left[P_{+} \phi \Gamma_{5}+P_{-} \phi^{\dagger} \Gamma_{5}\right] \\
& \left.\times \frac{H+m a \Gamma_{5}}{H^{2}+\left(m a \Gamma_{5}\right)^{2}}\left[P_{+} \phi \Gamma_{5}+P_{-} \phi^{\dagger} \Gamma_{5}\right]\right\}
\end{aligned}
$$

It can be confirmed that only the combination of the form $\phi^{\dagger} \phi \phi^{\dagger} \phi$ is logarithmically divergent. We thus examine, for example,

$$
\begin{aligned}
& -\frac{(2 g a)^{4}}{8} \operatorname{Tr}\left\{P_{-} \phi^{\dagger} \Gamma_{5} \frac{H+m a \Gamma_{5}}{H^{2}+\left(m a \Gamma_{5}\right)^{2}} P_{+} \phi \Gamma_{5} \frac{H+m a \Gamma_{5}}{H^{2}+\left(m a \Gamma_{5}\right)^{2}}\right. \\
& \left.\times P_{-} \phi^{\dagger} \Gamma_{5} \frac{H+m a \Gamma_{5}}{H^{2}+\left(m a \Gamma_{5}\right)^{2}} P_{+} \phi \Gamma_{5} \frac{H+m a \Gamma_{5}}{H^{2}+\left(m a \Gamma_{5}\right)^{2}}\right\} \\
& =-\frac{(2 g a)^{4}}{8} \operatorname{Tr}\left\{P_{-} \phi^{\dagger} \frac{\Gamma_{5} H}{H^{2}+\left(m a \Gamma_{5}\right)^{2}} P_{+} \phi \frac{\Gamma_{5} H}{H^{2}+\left(m a \Gamma_{5}\right)^{2}}\right. \\
& \left.\times P_{-} \phi^{\dagger} \frac{\Gamma_{5} H}{H^{2}+\left(m a \Gamma_{5}\right)^{2}} P_{+} \phi \frac{\Gamma_{5} H}{H^{2}+\left(m a \Gamma_{5}\right)^{2}}\right\} \\
& =-\frac{(2 g a)^{4}}{16} \phi^{\dagger} \phi \phi^{\dagger} \phi \\
& \times \operatorname{Tr}\left\{\frac{H^{2}}{H^{2}+\left(m a \Gamma_{5}\right)^{2}} \frac{\Gamma_{5}^{2}}{H^{2}+\left(m a \Gamma_{5}\right)^{2}} \frac{H^{2}}{H^{2}+\left(m a \Gamma_{5}\right)^{2}} \frac{\Gamma_{5}^{2}}{H^{2}+\left(m a \Gamma_{5}\right)^{2}}\right\}
\end{aligned}
$$


where we set the external momenta at 0 to extract the divergent coefficient. We have two contributions of this combination, and thus the total coefficient is given by

$$
-\frac{(2 g a)^{4}}{16} \times 2 \times 4=-\frac{(2 g a)^{4}}{2}
$$

where the last factor 4 is from the trace over Dirac matrices.

It is confirmed that all the higher order couplings are finite.

\subsection{Scalar Contributions}

We next examine the scalar contributions to various scalar couplings on the basis of the potential

$$
V=g\left[F \Gamma \phi^{2}+\left(F \Gamma \phi^{2}\right)^{\dagger}\right]
$$

\subsubsection{Tadpole Contribution}

The tadpole is evaluated by

$$
\begin{aligned}
& 2 g\left[\langle F \Gamma \phi\rangle \phi+\left\langle F^{\dagger} \Gamma \phi^{\dagger}\right\rangle \phi^{\dagger}\right] \\
& =-2 g \int\left[\phi \frac{a^{2} m \Gamma^{2}}{H^{2}+(a m \Gamma)^{2}}+\phi^{\dagger} \frac{a^{2} m \Gamma^{2}}{H^{2}+(a m \Gamma)^{2}}\right] \\
& =-2 m g a^{2}\left(\phi+\phi^{\dagger}\right) \int \frac{\Gamma^{2}}{H^{2}+(a m \Gamma)^{2}}
\end{aligned}
$$

which is precisely what we need to cancel the fermion contribution (4.2).

\subsubsection{Self-energy for Scalar Particles}

The one-loop self-energy of scalar particles is given by

$$
\begin{aligned}
& \frac{g^{2}}{2 !}\left[F \Gamma \phi^{2}+\left(F \Gamma \phi^{2}\right)^{\dagger}\right]\left[F \Gamma \phi^{2}+\left(F \Gamma \phi^{2}\right)^{\dagger}\right] \\
& \rightarrow \frac{g^{2}}{2 !}\left[4 \phi \phi\langle F \Gamma \phi\rangle\langle\Gamma \phi F\rangle+4 \phi^{\dagger} \phi^{\dagger}\left\langle F^{\dagger}(\Gamma \phi)^{\dagger}\right\rangle\left\langle(\Gamma \phi)^{\dagger} F^{\dagger}\right\rangle\right. \\
& \left.+8 \phi \phi^{\dagger}\left\langle F F^{\dagger}\right\rangle\left\langle\Gamma \phi(\Gamma \phi)^{\dagger}\right\rangle\right] \\
& =\frac{g^{2}}{2 !} \int\left[4 \phi \phi \frac{a^{2} m \Gamma^{2}}{H^{2}+(a m \Gamma)^{2}} \frac{a^{2} m \Gamma^{2}}{H^{2}+(a m \Gamma)^{2}}\right. \\
& +4 \phi^{\dagger} \phi^{\dagger} \frac{a^{2} m \Gamma^{2}}{H^{2}+(a m \Gamma)^{2}} \frac{a^{2} m \Gamma^{2}}{H^{2}+(a m \Gamma)^{2}} \\
& \left.+8 \phi \phi^{\dagger} \frac{-H^{2}}{H^{2}+(a m \Gamma)^{2}} \frac{a^{2} \Gamma^{2}}{H^{2}+(a m \Gamma)^{2}}\right] \\
& =-4 g^{2} a^{2} \phi \phi^{\dagger} \int \frac{\Gamma^{2}}{H^{2}+(a m \Gamma)^{2}}
\end{aligned}
$$




$$
\begin{aligned}
& +\frac{(m g)^{2} a^{4}}{2 !} \int\left[8 \phi \phi^{\dagger} \frac{\Gamma^{2}}{H^{2}+(a m \Gamma)^{2}} \frac{\Gamma^{2}}{H^{2}+(a m \Gamma)^{2}}\right. \\
& +4 \phi^{\dagger} \phi^{\dagger} \frac{\Gamma^{2}}{H^{2}+(a m \Gamma)^{2}} \frac{\Gamma^{2}}{H^{2}+(a m \Gamma)^{2}} \\
& \left.+4 \phi \phi \frac{\Gamma^{2}}{H^{2}+(a m \Gamma)^{2}} \frac{\Gamma^{2}}{H^{2}+(a m \Gamma)^{2}}\right] .
\end{aligned}
$$

The first term is quadratically divergent, and the remaining 3 terms are logarithmically divergent. These terms, when evaluated at vanishing external momenta, precisely cancel the fermion contributions (4.4).

\subsubsection{Triple Couplings}

The contributions from scalar one-loop diagrams to scalar triple couplings are generated by

$$
\frac{g^{3}}{3 !}\left\{\left[F \Gamma \phi^{2}+\left(\phi^{\dagger}\right)^{2} \Gamma F^{\dagger}\right]\left[F \Gamma \phi^{2}+\left(\phi^{\dagger}\right)^{2} \Gamma F^{\dagger}\right]\left[F \Gamma \phi^{2}+\left(\phi^{\dagger}\right)^{2} \Gamma F^{\dagger}\right]\right\}
$$

It is confirmed that the combinations $\phi \phi \phi$ and $\phi^{\dagger} \phi^{\dagger} \phi^{\dagger}$ are convergent. On the other hand, the combinations $\phi^{\dagger} \phi \phi$ and $\phi^{\dagger} \phi^{\dagger} \phi$ are logarithmically divergent. We thus set the external momenta at 0 to extact the coefficients of divergent terms. For example, we have

$$
\begin{aligned}
& \frac{g^{3}}{3 !}\left[\left(\phi^{\dagger}\right)^{2} \Gamma F^{\dagger}\right]\left[F \Gamma \phi^{2}\right]\left[F \Gamma \phi^{2}\right] \\
& \rightarrow \frac{g^{3}}{3 !}\left[16 \phi^{\dagger} \phi \phi\left\langle F \Gamma \Gamma F^{\dagger}\right\rangle\langle F \Gamma \phi\rangle\left\langle\phi \phi^{\dagger}\right\rangle\right] \\
& =\frac{16 g^{3}}{3 !} \phi^{\dagger} \phi \phi \int\left[\frac{-\Gamma^{2} H^{2}}{H^{2}+(m a \Gamma)^{2}} \frac{-m a^{2} \Gamma^{2}}{H^{2}+(m a \Gamma)^{2}} \frac{a^{2}}{H^{2}+(m a \Gamma)^{2}}\right] \\
& =\frac{16 g^{3} m a^{4}}{3 !} \phi^{\dagger} \phi \phi \int\left[\frac{H^{2}}{H^{2}+(m a \Gamma)^{2}} \frac{\Gamma^{2}}{H^{2}+(m a \Gamma)^{2}} \frac{\Gamma^{2}}{H^{2}+(m a \Gamma)^{2}}\right] .
\end{aligned}
$$

Since we have 3 terms of this combination, we have the numerical coefficient

$$
\frac{16 g^{3} m a^{4}}{3 !} \times 3=\frac{48 g^{3} m a^{4}}{3 !}
$$

which precisely cancels the fermion contribution (4.9), as required.

\subsubsection{Quartic Couplings}

The contributions from scalar one-loop diagrams to quartic couplings are generated by

$$
\begin{aligned}
& \frac{g^{4}}{4 !}\left[F \Gamma \phi^{2}+\left(\phi^{\dagger}\right)^{2} \Gamma F^{\dagger}\right]\left[F \Gamma \phi^{2}+\left(\phi^{\dagger}\right)^{2} \Gamma F^{\dagger}\right] \\
& \left.\times\left[F \Gamma \phi^{2}+\left(\phi^{\dagger}\right)^{2} \Gamma F^{\dagger}\right]\left[F \Gamma \phi^{2}+\left(\phi^{\dagger}\right)^{2} \Gamma F^{\dagger}\right]\right\} .
\end{aligned}
$$


It is confirmed that only the combination $\phi^{\dagger} \phi^{\dagger} \phi \phi$ is logarithmically divergent. We thus

set the external momenta at 0 to extact the coefficients of divergent terms. We thus have

$$
\begin{aligned}
& \frac{g^{4}}{4 !} \frac{4 \times 3}{2 !} \times 2^{4} \times 2 \phi^{\dagger} \phi^{\dagger} \phi \phi\left[\left\langle F \Gamma \Gamma F^{\dagger}\right\rangle\left\langle\phi \phi^{\dagger}\right\rangle\left\langle F \Gamma \Gamma F^{\dagger}\right\rangle\left\langle\phi \phi^{\dagger}\right\rangle\right] \\
& =\frac{g^{4}}{4 !} \frac{4 \times 3}{2 !} \times 2^{4} \times 2 \phi^{\dagger} \phi^{\dagger} \phi \phi \\
& \times \int\left[\frac{-\Gamma^{2} H^{2}}{H^{2}+(m a \Gamma)^{2}} \frac{a^{2}}{H^{2}+(m a \Gamma)^{2}} \frac{-\Gamma^{2} H^{2}}{H^{2}+(m a \Gamma)^{2}} \frac{a^{2}}{H^{2}+(m a \Gamma)^{2}}\right] \\
& =\frac{(g a)^{4}}{4 !} \frac{4 \times 3}{2 !} \times 2^{4} \times 2 \phi^{\dagger} \phi^{\dagger} \phi \phi \\
& \times \int\left[\frac{H^{2}}{H^{2}+(m a \Gamma)^{2}} \frac{\Gamma^{2}}{H^{2}+(m a \Gamma)^{2}} \frac{H^{2}}{H^{2}+(m a \Gamma)^{2}} \frac{\Gamma^{2}}{H^{2}+(m a \Gamma)^{2}}\right]
\end{aligned}
$$

where the last numerical coefficient 2 comes from the two possible ways to have $\left\langle F \Gamma \Gamma F^{\dagger}\right\rangle$. The numerical coefficient

$$
\frac{(g a)^{4}}{4 !} \frac{4 \times 3}{2 !} \times 2^{4} \times 2=\frac{(2 g a)^{4}}{2 !}
$$

is precisely what we need to cancel the fermion contribution (4.12).

It is confirmed that all the higher order couplings are finite.

\subsection{Fermion Self-energy Correction}

The self-energy correction to the fermion for general external momentum is given by

$$
\begin{aligned}
& \frac{1}{2 !}\left[2 g \bar{\psi}\left(P_{+} \phi \hat{P}_{+}+P_{-} \phi^{\dagger} \hat{P}_{-}\right) \psi\right]\left[2 g \bar{\psi}\left(P_{+} \phi \hat{P}_{+}+P_{-} \phi^{\dagger} \hat{P}_{-}\right) \psi\right] \\
& \rightarrow \frac{2(2 g)^{2}}{2 !} \bar{\psi} P_{+}\left[\int \hat{P}_{+} \frac{-1}{D+m \gamma_{5} \Gamma_{5}} P_{-} \frac{a^{2}}{H^{2}+(a m \Gamma)^{2}}\right] \hat{P}_{-} \psi \\
& +\frac{2(2 g)^{2}}{2 !} \bar{\psi} P_{-}\left[\int \hat{P}_{-} \frac{-1}{D+m \gamma_{5} \Gamma_{5}} P_{+} \frac{a^{2}}{H^{2}+(a m \Gamma)^{2}}\right] \hat{P}_{+} \psi \\
& =-4 g^{2} a^{3} \bar{\psi} P_{+}\left[\int \Gamma_{5} \frac{H+a m \Gamma_{5}}{H^{2}+\left(a m \Gamma_{5}\right)^{2}} \frac{1}{H^{2}+(a m \Gamma)^{2}}\right] P_{-} \Gamma_{5} \psi \\
& +4 g^{2} a^{3} \bar{\psi} P_{-}\left[\int \Gamma_{5} \frac{H+a m \Gamma_{5}}{H^{2}+\left(a m \Gamma_{5}\right)^{2}} \frac{1}{H^{2}+(a m \Gamma)^{2}}\right] P_{+} \Gamma_{5} \psi \\
& =-4 g^{2} a^{3} \bar{\psi} P_{+}\left[\int \frac{\Gamma_{5} H}{H^{2}+\left(a m \Gamma_{5}\right)^{2}} \frac{1}{H^{2}+(a m \Gamma)^{2}}\right] P_{-} \Gamma_{5} \psi \\
& +4 g^{2} a^{3} \bar{\psi} P_{-}\left[\int \frac{\Gamma_{5} H}{H^{2}+\left(a m \Gamma_{5}\right)^{2}} \frac{1}{H^{2}+(a m \Gamma)^{2}}\right] P_{+} \Gamma_{5} \psi
\end{aligned}
$$

where we used the fact that $H^{2}$ and $\Gamma_{5}^{2}$ are independent of $\gamma$ matrices and $P_{ \pm} \hat{P}_{ \pm}= \pm P_{ \pm} \Gamma_{5}$ for the external lines. 
By noting the relations

$$
P_{ \pm} \Gamma_{5} H P_{\mp}=P_{ \pm} \gamma_{5} H P_{\mp}= \pm P_{ \pm} H P_{\mp}
$$

the above amplitude is written as

$$
\begin{aligned}
& -4 g^{2} a^{3} \bar{\psi} P_{+}\left[\int \frac{H}{H^{2}+\left(a m \Gamma_{5}\right)^{2}} \frac{1}{H^{2}+(a m \Gamma)^{2}}\right] P_{-} \Gamma_{5} \psi \\
& -4 g^{2} a^{3} \bar{\psi} P_{-}\left[\int \frac{H}{H^{2}+\left(a m \Gamma_{5}\right)^{2}} \frac{1}{H^{2}+(a m \Gamma)^{2}}\right] P_{+} \Gamma_{5} \psi
\end{aligned}
$$

Because of the projection operators $P_{ \pm}$, which sandwich the amplitude with integral over the loop momentum, only the amplitude linear in $\gamma^{\mu}$ matrices survives the projection. We

thus have no terms corresponding to the mass term. The mass renormalization vanishes when renormalized at vanishing external momentum.

\subsection{Correction to Fermionic Vertex}

The one-loop correction to the fermionic vertex is finite as is seen in , for example,

$$
\begin{aligned}
& (2 g)^{3} a^{4} \bar{\psi} P_{+}\left[\int \hat{P}_{+} \frac{H+m a \Gamma_{5}}{H^{2}+\left(m a \Gamma_{5}\right)^{2}} \gamma_{5} P_{+} \phi \hat{P}_{+} \frac{H+m a \Gamma_{5}}{H^{2}+\left(m a \Gamma_{5}\right)^{2}} \gamma_{5} P_{-} \frac{1}{H^{2}+(m a \Gamma)^{2}}\right] \hat{P}_{-} \psi \\
& =(2 g)^{3} a^{4} \bar{\psi} P_{+}\left[\int \Gamma_{5} \frac{H+m a \Gamma_{5}}{H^{2}+\left(m a \Gamma_{5}\right)^{2}} \gamma_{5} P_{+} \phi \Gamma_{5} \frac{H+m a \Gamma_{5}}{H^{2}+\left(m a \Gamma_{5}\right)^{2}} \gamma_{5} P_{-} \frac{1}{H^{2}+(m a \Gamma)^{2}}\right] \hat{P}_{-} \psi \\
& =-(2 g)^{3} m a^{5} \bar{\psi} P_{+}\left[\int \frac{\Gamma_{5}^{2}}{H^{2}+\left(m a \Gamma_{5}\right)^{2}} P_{+} \phi \frac{\Gamma_{5} H}{H^{2}+\left(m a \Gamma_{5}\right)^{2}} P_{-} \frac{1}{H^{2}+(m a \Gamma)^{2}}\right] \hat{P}_{-} \psi \\
& =-(2 g)^{3} m a^{5} \bar{\psi} P_{+}\left[\int \frac{\Gamma_{5}^{2}}{H^{2}+\left(m a \Gamma_{5}\right)^{2}} P_{+} \phi \frac{H}{H^{2}+\left(m a \Gamma_{5}\right)^{2}} P_{-} \frac{1}{H^{2}+(m a \Gamma)^{2}}\right] \hat{P}_{-} \psi
\end{aligned}
$$

which is convergent and vanishes at vanishing external momenta after a symmetric integral over the internal momentum.

Our analysis so far establishes the non-renormalization (i.e., the absence of even finite renormalization) of the mass and interaction terms to the one-loop order in perturbation theory for a finite lattice spacing $a$ when renormalized at vanishing momenta.

\subsection{Renormalization of Kinetic Terms}

The one-loop correction to the "kinetic term" $F F^{\dagger}$ is given by

$$
\begin{aligned}
& \frac{g^{2}}{2 !}\left[F \Gamma(\phi)^{2}+\left(F \Gamma(\phi)^{2}\right)^{\dagger}\right]^{2} \\
& \rightarrow \frac{g^{2}}{2 !}\left[4 F \Gamma\left\langle\phi \phi^{\dagger}\right\rangle\left\langle\phi \phi^{\dagger}\right\rangle \Gamma^{\dagger} F^{\dagger}\right] \\
& =2 g^{2} a^{4} F \Gamma\left[\int \frac{1}{H^{2}+(a m \Gamma)^{2}} \frac{1}{H^{2}+(a m \Gamma)^{2}}\right] \Gamma F^{\dagger}
\end{aligned}
$$


which is logarithmically divergent.

The correction to the kinetic term of scalar particles is given in (4.3)

$$
g^{2} a^{2} \operatorname{Tr} \phi^{\dagger} \frac{H \Gamma_{5}}{H^{2}+\left(m a \Gamma_{5}\right)^{2}} \phi \frac{\Gamma_{5} H}{H^{2}+\left(m a \Gamma_{5}\right)^{2}}
$$

where the quadratic divergence evaluated at vanishing external momentum is cancelled by the scalar loop diagrams.

The fermion self-energy correction is given by (4.24)

$$
\begin{aligned}
& -4 g^{2} a^{3} \bar{\psi} P_{+}\left[\int \frac{H}{H^{2}+\left(a m \Gamma_{5}\right)^{2}} \frac{1}{H^{2}+(a m \Gamma)^{2}}\right] P_{-} \Gamma_{5} \psi \\
& -4 g^{2} a^{3} \bar{\psi} P_{-}\left[\int \frac{H}{H^{2}+\left(a m \Gamma_{5}\right)^{2}} \frac{1}{H^{2}+(a m \Gamma)^{2}}\right] P_{+} \Gamma_{5} \psi
\end{aligned}
$$

We have to examine if a universal wave function renormalization is sufficient to remove the divergence from these 3 contributions.

One can in fact show that a uniform subtraction of logarithmic infinity renders all these expressions finite. The integral for $F F^{\dagger}$ (4.26) is written in more detail as

$$
2 g^{2} \Gamma(a p)\left[\int_{-\pi}^{\pi} \frac{d^{4} k}{(2 \pi)^{4}} \frac{1}{H^{2}(k+a p)+(a m \Gamma)^{2}(k+a p)} \frac{1}{H^{2}(k)+(a m \Gamma)^{2}(k)}\right] \Gamma(a p) .
$$

where we chose the basic Brillouin zone at $\frac{-\pi}{a}<k_{\mu} \leq \frac{\pi}{a}$ and rescaled the integration variables as $a k_{\mu} \rightarrow k_{\mu}$. If one renormalizes this expression at $p_{\mu}=0$, the wave function renormalization factor is defined by

$$
Z=1-2 g^{2} \int_{-\pi}^{\pi} \frac{d^{4} k}{(2 \pi)^{4}} \frac{1}{H^{2}(k)+(a m \Gamma)^{2}(k)} \frac{1}{H^{2}(k)+(a m \Gamma)^{2}(k)}
$$

by noting $\Gamma(0)=1$. This integral in $Z$ is logarithmically divergent for $a \rightarrow 0$ : The divergence appears as an infra-red divergence. The divergent part in (4.26) at vanishing external momentum is extracted by considering small $\delta,|\delta| \ll 1$, as

$$
\begin{aligned}
& 2 g^{2} \int_{-\delta}^{\delta} \frac{d^{4} k}{(2 \pi)^{4}} \frac{1}{H^{2}(k)+(a m \Gamma)^{2}(k)} \frac{1}{H^{2}(k)+(a m \Gamma)^{2}(k)} \\
& \simeq 2 g^{2} \int_{-\delta}^{\delta} \frac{d^{4} k}{(2 \pi)^{4}} \frac{1}{k^{2}+(a m)^{2}} \frac{1}{k^{2}+(a m)^{2}} \\
& =2 g^{2} \int_{-\delta / a}^{\delta / a} \frac{d^{4} k}{(2 \pi)^{4}} \frac{1}{k^{2}+m^{2}} \frac{1}{k^{2}+m^{2}} .
\end{aligned}
$$

Note that $H^{2} \sim O(1)$ for the domain of species doublers and thus no infrared divergence.

The divergent part of the self-energy of scalar particles (4.27) is extracted by considering small $p_{\mu}$ and $\delta$ as

$$
\frac{g^{2}}{a^{2}} \operatorname{Tr} \int_{-\delta}^{\delta} \frac{d^{4} k}{(2 \pi)^{4}} \frac{H(k+a p) \Gamma_{5}(k+a p)}{H^{2}(k+a p)+\left(m a \Gamma_{5}\right)^{2}(k+a p)} \times \frac{\Gamma_{5}(k) H(k)}{H^{2}(k)+\left(m a \Gamma_{5}\right)^{2}(k)}
$$




$$
\begin{aligned}
& \simeq \frac{g^{2}}{a^{2}} \operatorname{Tr} \int_{-\delta}^{\delta} \frac{d^{4} k}{(2 \pi)^{4}} \frac{-\gamma_{5}(\not k+a \not p) \gamma_{5}}{(k+a p)^{2}+(m a)^{2}} \times \frac{-\not k}{k^{2}+(m a)^{2}} \\
& =4 \frac{g^{2}}{a^{2}} \int_{-\delta}^{\delta} \frac{d^{4} k}{(2 \pi)^{4}} \frac{k^{2}+a k p}{k^{2}+2 a k p+(a p)^{2}+(m a)^{2}} \times \frac{1}{k^{2}+(m a)^{2}} \\
& \rightarrow 4 \frac{g^{2}}{a^{2}} \int_{-\delta}^{\delta} \frac{d^{4} k}{(2 \pi)^{4}}\left[-\frac{\left(k^{2}+a k p\right)\left(2 a k p+(a p)^{2}\right)}{\left(k^{2}+(m a)^{2}\right)^{3}}\right. \\
& \left.+\frac{\left(k^{2}+a k p\right)\left(2 a k p+(a p)^{2}\right)^{2}}{\left(k^{2}+(m a)^{2}\right)^{4}}\right] \\
& =4 \frac{g^{2}}{a^{2}} \int_{-\delta}^{\delta} \frac{d^{4} k}{(2 \pi)^{4}}\left[-\frac{k^{2}(a p)^{2}+2(a k p)^{2}}{\left(k^{2}+(m a)^{2}\right)^{3}}+\frac{4 k^{2}(a k p)^{2}}{\left(k^{2}+(m a)^{2}\right)^{4}}\right] \\
& =4 g^{2} p^{2} \int_{-\delta}^{\delta} \frac{d^{4} k}{(2 \pi)^{4}}\left[-\frac{3}{2} \frac{k^{2}}{\left(k^{2}+(m a)^{2}\right)^{3}}+\frac{k^{4}}{\left(k^{2}+(m a)^{2}\right)^{4}}\right] \\
& \rightarrow 2 g^{2} \int_{-\delta}^{\delta} \frac{d^{4} k}{(2 \pi)^{4}} \frac{1}{\left(k^{2}+(m a)^{2}\right)^{2}} \times\left(-p^{2}\right)
\end{aligned}
$$

where we used the fact that the $p$-independent terms are cancelled by the mass correction arising from the scalar particle one-loop diagrams. We also took account of a symmetric integral over $k_{\mu}$. Note that our $\gamma^{\mu}$ matrices are anti-hermitian, and $k^{2} \geq 0$. We thus have the same divergent part as that of the $F F^{\dagger}$ term in (4.31).

Similarly, the divergent part of the fermion self-energy correction (4.28) is extracted by considering small $p_{\mu}$ and $\delta$.

$$
\begin{aligned}
& -4 \frac{g^{2}}{a} P_{+}\left[\int_{-\delta}^{\delta} \frac{d^{4} k}{(2 \pi)^{4}} \frac{H(k+a p)}{H^{2}(k+a p)+\left(a m \Gamma_{5}\right)^{2}(k+a p)} \times \frac{1}{H^{2}(k)+(a m \Gamma)^{2}(k)}\right] \\
& \times P_{-} \Gamma_{5}(a p) \\
& -4 \frac{g^{2}}{a} P_{-}\left[\int_{-\delta}^{\delta} \frac{d^{4} k}{(2 \pi)^{4}} \frac{H(k+a p)}{H^{2}(k+a p)+\left(a m \Gamma_{5}\right)^{2}(k+a p)} \times \frac{1}{H^{2}(k)+(a m \Gamma)^{2}(k)}\right] \\
& \times P_{+} \Gamma_{5}(a p) \\
& \simeq-4 \frac{g^{2}}{a} \int_{-\delta}^{\delta} \frac{d^{4} k}{(2 \pi)^{4}} \frac{-\gamma_{5}(\not k+a \not p)}{(k+a p)^{2}+(a m)^{2}} \times \frac{1}{k^{2}+(a m)^{2}} \gamma_{5} \\
& \rightarrow-4 \frac{g^{2}}{a} \int_{-\delta}^{\delta} \frac{d^{4} k}{(2 \pi)^{4}}\left[\frac{a \not p}{\left(k^{2}+(a m)^{2}\right)^{2}}-\frac{1}{2} \frac{a \not k^{2}}{\left(k^{2}+(a m)^{2}\right)^{3}}\right] \\
& \rightarrow 2 g^{2} \int_{-\delta}^{\delta} \frac{d^{4} k}{(2 \pi)^{4}} \frac{1}{\left(k^{2}+(a m)^{2}\right)^{2}} \times(-\not p)
\end{aligned}
$$

by taking into account a symmetric integral over $k_{\mu}$. We thus obtain the same divergent part as the other two wave function renormalization factors (4.31) and (4.32).

The finite part in wave function renormalization is, however, generally different for these three quantities, which will cause a deviation from supersymmetry, such as the quadratic divergence of the scalar mass correction, in the two-loop order in perturbation theory. 


\section{Discussion and Conclusion}

We have studied the implications of lattice chiral symmetry satisfied by the GinspargWilson operators when applied to a regularization of the supersymmetric Wess-Zumino model. We found a conflict between the lattice chiral symmetry and the Euclidean Majorana condition for Yukawa couplings. (In the Weyl representation, we recognized a conflict between the lattice chiral symmetry and Yukawa couplings.) We thus have basically two alternative choices: the first one is to impose lattice chiral symmetry but sacrifice the Euclidean Majorana condition, and the second one is to sacrifice lattice chiral symmetry but preserve the Euclidean Majorana condition. The latter approach is similar to the analysis of Bartels and Kramer [17 on the basis of the conventional Wilson fermion, where one-loop level non-renormalization of the superpotential has been shown: We have also confirmed this property for the Lagrangian (2.28). In this paper, we examined in detail the case (2.17) where the lattice chiral symmetry together with naive Bose-Fermi symmetry is imposed. This scheme incorporates a SUSY-like symmetry in the free part of the Lagrangian and preserves one-loop level non-renormalization of the superpotential for finite lattice spacing, independently of the parameter $k$ in (1.2) and independently of the detailed construction of lattice Dirac operators. Both schemes however fail in preserving the uniform wave function renormalization for all the field variables. This generally causes the failure in the cancellation of quadratic divergences in the two-loop order in perturbation theory. These two schemes are thus on a similar footing.

From a view point of practical diagramatical calculations, our analysis illustrates a transparent treatment of various diagrams on the basis of algebraic properties without recourse to the detailed construction of lattice Dirac operators. The actual calculations are almost identical to those in continuum theory, and the power counting of various daigrams agrees with that of the continuum theory, as was shown in Section 3; this agreement of the power counting does not hold for the Lagrangian (2.28). Although our analysis is perturbative, a perturbative lattice analysis of Yukawa couplings is expected to be important when one applies a non-perturbative analysis to the gauge theoretical sector of a supersymmetric model of elementary particles, just to preserve the consistency of various sectors of a single model. When one includes a background gauge field into our treatment, one need to covariantize the operator $D^{\dagger} D$ in the bosonic sector by a lattice analogue of

$$
\partial_{\mu} \rightarrow \partial_{\mu}-i g A_{\mu}
$$

independently円 of the fermionic sector defined by $D$, to avoid the appearance of the Pauli term, for example, in $D^{\dagger} D$.

There exists an argument for non-renormalization theorem on the basis of the stability of supersymmetry and holomorphic properties 28. The holomorphic properties are partly related to chiral symmetry and partly related to a clear separation between the kinetic (Kähler) and superpotential terms. Our analysis indicates that these two requirements

\footnotetext{
${ }^{9}$ Note that $D^{\dagger} D$ after covariantizing $D$ and a direct covariantization of $D^{\dagger} D$ are generally different. We also note that the locality of $D$ for $k>0$ with dynamical gauge field has not been established yet 23.
} 
are in conflict in the lattice regularization known so fart bra, it is known that the Leibniz rule generally fails in the lattice difference operation 16. Apparently, much need to be learned before we obtain a coherent picture of the lattice regularization of supersymmetry.

We thank H. So and H. Suzuki for helpful comments.

\section{References}

[1] J. Wess and J. Bagger, Supersymmetry and Supergravity (Princeton Univ. Press, 1983).

S. Weinberg, Quantum Theory of Fields III (Cambridge Univ. Press, 2000).

[2] J. Wess and B. Zumino, Phys. Lett. 49B(1974)52.

[3] J. Iliopoulos and B. Zumino, Nucl. Phys. B76 (1974)310.

[4] K. Fujikawa and W. Lang, Nucl. Phys. B88 (1975) 61.

P. West,Nucl. Phys. B106 (1976) 219.

M.T. Grisaru, M. Rocek and W. Siegel, Nucl. Phys. B159 (1979) 429.

[5] P.H. Ginsparg and K.G. Wilson, Phys. Rev. D25 (1982)2649.

[6] H. Neuberger, Phys. Lett.417B(1998)141;427B(1998)353.

[7] P. Hasenfratz, V. Laliena and F. Niedermayer, Phys. Lett. 427B(1998)125.

[8] M. Lüscher, Phys. Lett. 428B(1998)342.

[9] P. Hasenfratz, Nucl. Phys. B525 (1998) 401.

[10] P. Hernandez, K. Jansen and M. Lüscher, Nucl. Phys. B552 (1999)363.

[11] H. Neuberger, Phys.Rev.D61(2000)085015.

[12] I. Horvath, Phys. Rev. Lett. 81 (1998) 4063.

[13] F. Niedermayer, Nucl. Phys, Proc. Suppl.73 (1999) 105.

H. Neuberger, "Exact chiral symmetry on the lattice," hep-lat/0101006.

M. Lüscher, "Chiral gauge theories revisited," hep-th/0102028.

M. Creutz, "Aspects of chiral symmetry and the lattice," hep-lat/0007032.

[14] T. Aoyama and Y. Kikukawa, Phys. Rev. D59(1999)054507.

H. So and N. Ukita, Phys. Lett. 457B(1999)314; Nucl. Phys. Suppl. 94 (2001) 795.

\footnotetext{
${ }^{10}$ For the Lagrangian (2.28), the term $\mathcal{L}_{M(2)}$ pot $(2.32)$ breaks $U(1)$ in $U(1) \times U(1)_{R}$ symmetry even for $m=g=0$ and thus the argument in [28] does not work.
} 
[15] W. Bietenholz, Mod. Phys. Lett. A14(1999)51.

S. Catterall and S. Karamov, hep-lat/0108024.

[16] P. Dondi and H. Nicolai, Nuovo Cim. A41 (1977) 1.

[17] J. Bartels and G. Kramer, Z. Phys. C20(1983) 159.

[18] S. Elitzur, E.Ravinovici and A. Schwimmer, Phys. Lett. 119B (1982) 165.

N. Sakai and M. Sakamoto, Nucl. Phys. B229 (1983) 173.

J. Bartels and J. B. Bronzan, Phys. Rev. D28 (1983) 818.

S. Nojiri, Prog. Theor. Phys. 74 (1985) 819;74 (1985) 1124.

[19] T. Banks and P. Windy, Nucl. Phys. B198(1982) 226.

I. Ichinose, Phys. Lett. 122B (1983) 68.

R. Nakayama and Y. Okada, Phys. Lett. 134B (1984) 241.

G. Curci and G. Veneziano, Nucl. Phys. B292(1987) 555.

I. Montvay, "SYM on the lattice," hep-lat/9801023 and references therein.

[20] R. Narayanan and H. Neuberger, Nucl. Phys. B443(1995)305.

S. Aoki, K. Nagai and S.V. Zenkin, Nucl. Phys. B508 (1997) 715.

N. Maru and J. Nishimura, Int. J. Mod. Phys. A13 (1998) 2841.

R.G. Edwards, U.M. Heller and R. Narayanan, Chin. J. Phys. 38(2000) 594.

D. B. Kaplan and M. Schmaltz, Chin. J. Phys. 38(2000) 543.

G. T. Fleming, J. B. Kogut and P. M. Vranas, Phys. Rev. D64 (2001) 034510, and references therein.

K. Itoh, M. Kato, H.So, H. Sawanaka, and N. Ukita, "Super Yang-Mills theory on lattice and the transformation", talk given at Lattice 2001, Berlin.

[21] K. Fujikawa, Nucl. Phys. B589(2000)487.

K. Fujikawa and M. Ishibashi, Nucl. Phys. B587(2000)419.

[22] S. Chandrasekharan, Phys. Rev. D60 (1999) 074503.

[23] K. Fujikawa and M. Ishibashi, Nucl. Phys. B605(2001) 365.

[24] T.W. Chiu, Nucl. Phys. B588 (2000) 400; Phys. Lett. 498B (2001) 111; Nucl. Phys. Proc. Suppl.94 (2001) 733.

[25] H. Nicolai, Nucl. Phys.B140 (1978) 294; B156 (1979) 157; B156 (1979) 177.

[26] P. van Nieuwenhuizen and A. Waldron, Phys. Lett.397B(1996) 29, and references therein.

[27] H. Suzuki, JHEP 0010(2000)039.

[28] N. Seiberg, Phys. Lett. 318B (1993) 469. 